\title{
An overview of the management of back pain
}

\author{
N Padayachee, ${ }^{1,3}$ M Lundie, $^{2}$ N Schellack ${ }^{2}$ \\ ${ }^{1}$ Department of Pharmacy and Pharmacology, University of Witwatersrand. \\ ${ }^{2}$ School of Pharmacy, Faculty of Health Sciences, Sefako Makgatho Health Sciences University \\ ${ }^{3}$ Faculty of Health Sciences, School of Therapeutic Sciences \\ ${ }^{*}$ Corresponding author, email: Neelaveni.padayachee@wits.ac.za
}

\begin{abstract}
Back pain affects people across any socio-economic category and is a leading cause of absenteeism and decreased productivity in the workplace. This symptomatic condition is caused by multiple factors, making it difficult to manage. With a small proportion of people experiencing back pain due to pathological reasons, in the larger majority, back pain is due to a mechanical cause. Taking a complete history that includes identifying risk factors such as depression, poor posture, lack of exercise, older age and a physically demanding job is crucial to the effective management of the condition. Behavioural, psychological and social factors of the patient should form the backbone for treatment of back pain. Non-pharmacological management such as exercise, spinal manipulation and acupuncture should be the first-line treatment; however, if this provides poor results then pharmacological measures such as the use of non-steroidal anti-inflammatory drugs (NSAIDS) like ibuprofen or muscle relaxants such as cyclobenzaprine should be considered.
\end{abstract}

Keywords: back pain, narcotics, non-narcotics, NSAIDs

\section{Key Summary Points}

- Back pain is the sixth leading complaint at a primary health care level.

- Back pain is largely as a result of mechanical causes, such as physical factors, psychological factors or a combination of both.

- A smaller percentage $(<1 \%)$ of back pain is caused by pathological reasons, such as malignancy, infection or vertebral fracture.

- Risk factors for back pain include physically demanding jobs, physical comorbidities, depression, smoking, obesity, lack of exercise, awkward postures, feeling tired, female gender, desk bound jobs and older age.

- Optimal management includes non-pharmacological management (PRICE, TEN) and pharmacological management (analgesics, narcotics, non-narcotics).

\section{Introduction}

Pain is defined as "an unpleasant sensory and emotional experience associated with actual or potential tissue damage or described in terms of such damage", however, it is largely subjective and varies from patient to patient. ${ }^{1}$ It is reported that more than $80 \%$ of people experience back pain at a point in their lives. ${ }^{2}$ In South Africa, back pain is the sixth most common complaint in the primary health care setting. ${ }^{3}$ It is therefore not surprising that back pain is the sixth leading cause contributing to the global disease burden, when one considers disease burden with regard to disability-adjusted life years. ${ }^{4}$ However, back pain is, as a symptom, caused by various factors, and not a disease itself, which explains the limited and ineffective prevention strategies compiled to date. ${ }^{3,4,5}$

\section{Prevalence of back pain}

Low back pain is a major public health problem affecting men and women from high-income, middle-income and low-income countries. ${ }^{3,6,7}$ The Global Burden of Disease Study identified back pain as the leading cause of disability globally.,7 Certain studies found that back pain is most prevalent in ages 30 to 69 years and stated that the female gender is more often affected. $3,5,8,9$ However, gender pattern may be reversed in lower-income countries and back pain is reported mostly amongst men in Africa. They speculated that this may be due to the African culture, where men perform intensive physical labour and where there may be under-reporting of back pain by women due to possible gender inequalities. ${ }^{3}$ Maher, Underwood and Buchbinder concluded that high-income countries have more reported cases of back pain compared to middle- and low-income countries. Contrarily, there is an inverse correlation between the prevalence of back pain and patients' educational status. There is also a logical connection with occupational factors, where the prevalence of back pain is increased in positions with high physical demands. ${ }^{2}$

\section{Cost and burden of back pain}

Back pain is reported as the major reason for occupational absenteeism, decreased work productivity and early retirement globally, which results in considerable economic burden to individuals and the wider public.,5,8,10 To put the issue into perspective: back pain is the chronic condition responsible for more people forced out of the workplace than cardiovascular disease, diabetes, cancer and respiratory conditions combined. ${ }^{5}$ 
Direct and indirect cost implications for back pain, including healthcare expenditure and disability insurance, contribute to the strain on global health-care costs. ${ }^{5,6,8,11}$ In the United States, lower back pain results in 34 million appointments with family physicians and primary care internists annually, leading to a total of 50 billion dollars in direct costs. ${ }^{12}$ The prediction for the coming decades is that disability and economic impact related to back pain will increase in especially low- and middle-income countries (e.g. Asia, Africa and the Middle East), as health and social systems are not well established and are too ill-equipped to handle this burden along with the growing prevalence of infectious diseases. ${ }^{3}$

\section{Pathophysiology of back pain}

Anatomically, back pain is described as pain localised from below the shoulder blades up to the gluteal folds and can involve muscles, ligaments, facet joints, spinal nerve roots, vertebral periosteum, intervertebral discs and blood vessels. Pain localised around the lumbosacral vertebra and iliac crests (from the twelfth rib to the coccyx) is known as lower back pain and is the most common form of back pain. ${ }^{13}$ There are five lumbar vertebrae located in the lower back, separated by intervertebral discs and the foramina, which accommodate the spinal nerves, radicular blood vessels and the sinuvertebral nerves. The spine has weightbearing and shock-absorbing functions and is responsible for the protection of the spinal cord and nerve roots. The ligaments, facet joints and muscles surrounding the spine control balance, flexibility and stability. ${ }^{8}$ Back pain can arise from mechanical disorders of the spine (e.g. disc protrusion or spinal stenosis), non-mechanical diseases of the spine (e.g. cancer or infection) or referred pain from urogenital or gastrointestinal diseases. The ligaments of the intervertebral disc complex, facet joints and paravertebral musculature are supplied with nociceptive fibres, i.e. pain receptors. It is important to distinguish between pain in the spine itself and muscular pain, i.e. myofascial pain, as treatment strategies need to focus on the presumed source of the pain. ${ }^{13}$

Acute pain acts as a physiological warning by the body of the presence of disease or a harmful condition. ' Further to this, acute lower back pain generally arises from increased tension in the muscles surrounding the spine with resulting tearing, either of the muscle fibres themselves or of the tendons between the muscles and bone.

Back pain that originates in the musculoskeletal system can be described in three categories ${ }^{13}$ :
- Muscle spasm: Muscle spasm is considered a protective measure in an untrained or poorly conditioned muscle, which becomes inflamed due to persistent overuse. The surrounding muscles contract to prevent further movement in the inflamed area, which will worsen the pain. 8,13 Studies concluded that muscle spasm can also be caused by ischaemia in a specific muscle rather than muscle tension. ${ }^{13}$

- Myofascial pain: Myofascial pain results from overuse of or damage to muscle fibres that are then in a shortened or contracted state. Myofascial pain is characterised by localised, palpable trigger points, which cause radiating, aching-type pain upon palpation.

- Peripheral fatigue is the result of mononeuronal firing or muscle glycogen depletion that leads to impairment of the neuromusculoskeletal function in the paravertebral muscles. ${ }^{8,13}$

Acute back pain can result from ligament sprains as well, where the ligament is extended beyond its normal range. ${ }^{8}$

Acute pain that remains untreated or is treated inefficiently can result in the development of chronic pain. ${ }^{1}$ Acute low back pain becomes chronic when there is no relief for a period of more than three months. ${ }^{11}$ Chronic low back pain can be the result of serious disease or damage, but is mostly caused by deterioration of the bony structures and ligaments in the spine, which is considered a normal process with advanced age. The facet joints undergo degenerative or inflammatory changes, which result in the enlargement of these joints and therefore facetmediated arthritic pain. ${ }^{8}$ A chronic pain experience is not only physiological, but involves somatic, psychological and social factors, leading to pain, distress and disability in an individual ${ }^{10}$ and affects social, recreational and work life. ${ }^{11}$

\section{Causes of back pain}

The vast majority of people consulted for back pain in primary care settings are diagnosed with non-specific back pain, as there is no apparent specific nociceptive cause. ${ }^{3,5,7,9,14}$ Golob and Wipf stated that in $97 \%$ of patients who presented with back pain, the back pain had a mechanical cause. Physical factors (e.g. lifting a load awkwardly), psychological factors (e.g. feeling tired or overworked), or a combination of these factors (e.g. a distraction during hard physical labour) can result in the development of acute back pain, but the activity that caused the back pain cannot always be recalled by the patient. ${ }^{5}$ Studies found that the majority of activities resulting in back pain occurred early in the morning, due to the overnight absorption of fluid into

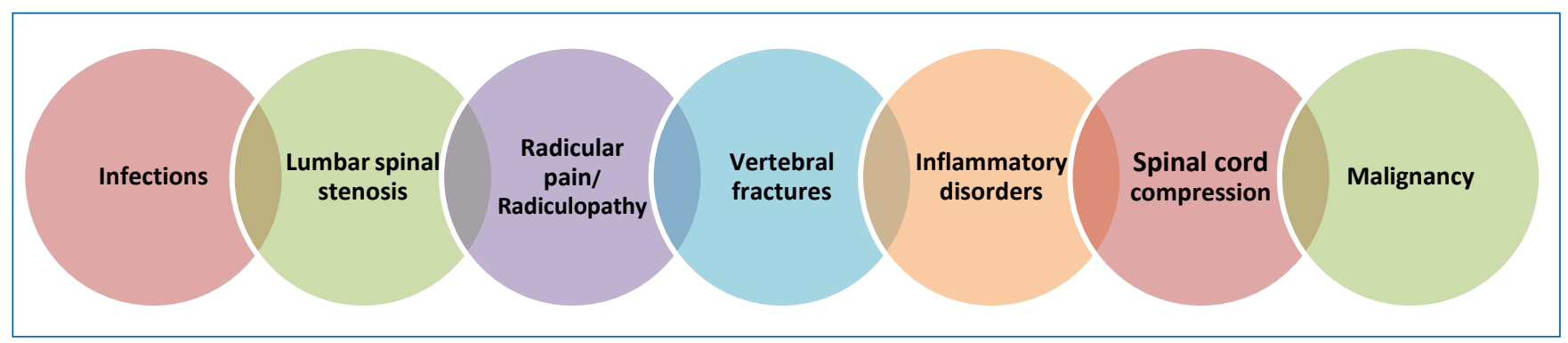

Figure 1: Pathological causes of back pain 
the intervertebral discs, which results in the discs being more sensitive to injury when put under stress. ${ }^{4,5}$ Maher and colleagues also found that the majority of back injuries occur at home and that the leading cause was heavy lifting.

A small proportion of back pain patients (less than $1 \%$ of patients presenting with back pain at primary care settings) can be accurately diagnosed with a known pathological cause, which are generally serious conditions, such as malignancy, infection and vertebral fracture, and require targeted treatment., ${ }^{3,14}$ Outlined briefly below (Figure 1) are some of the most common pathological causes of back pain:

- Radicular pain/Radiculopathy - There is nerve-root involvement, most commonly in the L5 and S1. Patients typically presents with leg pain which is worse than their back pain and which worsens further during coughing, sneezing, straining and straight leg raise tests. The most common cause is disc herniation or degenerative changes with local inflammation. $3,8,14$

- Lumbar spinal stenosis - Patients experience back pain, which spreads into one or both legs, while walking or during extended periods of standing. Relief is experienced during rest or lumbar flexion (leaning forward). The most common cause is degenerative changes due to facet osteoarthritis, ligamentum flavum hypertrophy and bulging discs, where the foramina is narrowed. $3,8,14$

- Vertebral fractures - These fractures are mainly caused by osteoporosis. The probability to develop vertebral fractures increases with the female gender, an age of 70 years or above, severe trauma and the chronic use of glucocorticoids. 3,14

- Inflammatory disorders (e.g. axial spondyloarthritis) Axial spondyloarthritis is a chronic inflammatory disease that most commonly develops in people aged 20 to 40 years. It is characterised by morning stiffness in the lower back, which improves with exercise but not with rest. These patients are commonly erroneously diagnosed with non-specific low back pain. $^{3}$

- Malignancy - Evidently, back pain will be a major symptom of myeloma, but metastatic cancer also commonly targets bone. The most common cancers that spread to bone are adenocarcinomas, e.g. breast, lung, prostate, thyroid and gastrointestinal cancers. ${ }^{3,14}$ The most common symptom of metastasis to the spine is sudden severe pain, but also neurological symptoms due to spinal cord compression or spinal instability. ${ }^{14}$

- Infections - Spondylodiscitis, vertebral osteomyelitis, epidural abscess and even bacterial infections, such as tuberculosis and brucellosis, may all result in back pain. A study that was done in Uganda highlighted the burden of infectious diseases in low-income countries, when back pain resulting from infections, such as tuberculosis and brucellosis, was found. Immunosuppressed patients and intravenous drug users have an increased risk for the development of spinal infections. ${ }^{3,14}$ Fever and malaise that occur initially are not specific and can lead to serious consequences if left untreated. ${ }^{14}$
- Spinal cord compression (cauda equina syndrome) - Cord compression is most commonly caused by the herniation of an intervertebral disc, which mainly occurs during metastases. Other symptoms are motor weakness and bowel and/or bladder dysfunction. ${ }^{14}$

\section{Risk factors}

Table 1 provides a checklist of risk factors that the health care worker can use to assess the likelihood for the development of back pain.

Table 1: Check list of risk factors for the development of back pain

\begin{tabular}{|c|c|}
\hline Risk Factor & Check $\checkmark$ \\
\hline $\begin{array}{l}\text { Physically demanding jobs - manual handling, bending, } \\
\text { twisting and whole-body vibration } 2,3,4,5,7,14\end{array}$ & \\
\hline Physical comorbidities, i.e. asthma, headache, diabetes ${ }^{3}$ & \\
\hline Depression $2,3,5,7,14$ & \\
\hline Smoking ${ }^{3,5,14}$ & \\
\hline Obesity $^{2,3,5,7,14}$ & \\
\hline Lack of exercise ${ }^{3}$ & \\
\hline Awkward postures ${ }^{3,4}$ & \\
\hline Feeling tired ${ }^{3,4}$ & \\
\hline Distractions during an activity 3,4 & \\
\hline Older age $\mathrm{e}^{2,7,14}$ & \\
\hline Female gender ${ }^{7,14}$ & \\
\hline Inactive/desk-bound work ${ }^{14}$ & \\
\hline Psychologically arresting work ${ }^{14}$ & \\
\hline Low educational status $2,7,14$ & \\
\hline Workers' compensation insurance ${ }^{14}$ & \\
\hline Job dissatisfaction ${ }^{2,14}$ & \\
\hline Insomnia ${ }^{9}$ & \\
\hline Anxiety/Stress ${ }^{2,4,9,14}$ & \\
\hline Handling of objects far from the body ${ }^{4}$ & \\
\hline Handling people or animals and unstable loading ${ }^{4}$ & \\
\hline A slip, trip or fall ${ }^{4}$ & \\
\hline Engagement in moderate or vigorous physical activity ${ }^{4}$ & \\
\hline Sexual activity ${ }^{4}$ & \\
\hline Alcohol consumption ${ }^{4}$ & \\
\hline
\end{tabular}

\section{Differential diagnosis of back pain}

The American College of Physicians and the American Pain Society recommend that low back pain be divided into three categories: non-specific low back pain, low back pain with potential radicular symptoms, or low back pain due to an identified spinal cause, e.g. metastasis or infection. ${ }^{2,15}$ Differential diagnosis is initiated with a thorough physical examination and extensive history taking to determine which patients need to be referred for imaging and further diagnostic evaluation. ${ }^{5,8,14}$ The majority of patients who present with acute back pain can be treated with first-line non-pharmacological or pharmacological therapy and do not require any further evaluation. ${ }^{14,15} \mathrm{~A}$ number of "red flags" have been identified to discern patients who require further diagnostic evaluation, e.g. night pain, fever, a history of malignancy, unintentional weight loss, prolonged use of corticosteroids, severe trauma, pain occurring for an extended 
period of longer than four weeks and the presence of contusion or abrasion. 5,14

\section{History taking}

History taking involves enquiries about the position, duration and severity of the back pain; whether the patient has suffered from back pain before and a comparison from the patient between the prior and the current back pain; as well as any event that may have triggered the onset of the pain. Unintentional weight loss, night fever or pain, changes in bowel and/or bladder function, or neurological symptoms, such as weakness, numbness and disequilibrium, also need to be determined. 2,8,14 Bladder or bowel dysfunction may indicate cauda equina syndrome, which is a serious medical emergency. ${ }^{8}$ A detailed history of any failed treatment for the back pain, malignancy, bacterial infections, use of injectable drugs, corticosteroid use and back procedures should be recorded. 2,8,14 The probability of spinal infection is increased with prior or current use of intravenous drugs, recent bacterial infections or if the patient has a fever. ${ }^{8,14}$ Mechanical back pain is generally located in the spine and may radiate to the gluteus, but does not spread into the legs, as is the case with radicular pain. ${ }^{8}$ An informal psychological evaluation to determine the presence of depression may be helpful, as psychological disorders are strong predictors of outcomes in terms of prolonged pain and disability course. ${ }^{8,14}$

\section{Physical examination}

A primary diagnosis cannot be the expected result of the physical examination, but the physical examination will contribute to the identification of factors that may suggest further evaluation. The patient's back and posture must be examined to evaluate range of motion and to identify abnormalities, such as scoliosis. The spine must be palpated to identify sensitive vertebrae or soft tissue, as well as the abdomen to evaluate referred pain from visceral organs; the palpation of potential areas of malignancy can also be included. $2,8,14$ The consultant must perform a neurological examination to evaluate reflexes, strength, sensation and stride. $^{2,14}$ Suspected radiculopathy can be identified with a straight leg raise test. ${ }^{8,14}$

\section{Imaging}

Plain-film radiography, magnetic resonance imaging (MRI) and computed tomography (CT) is reserved for patients with a high possibility of infection, fracture or cauda equina, where it is necessary to determine the exact pathological reason for back pain to facilitate surgery or other interventions. 2,14,15 Early and unjustifiable imaging do not result in improved outcomes as non-specific back pain generally improves within four to six weeks with or without treatment, but rather lead to unnecessary radiation exposure and increased patient anxiety, work absence, health-care costs and utilisation of invasive procedures. $2,5,8,14$ Guidelines from the American College of Physicians, the American Pain Society, the National Institute for Health and Care Excellence in the United Kingdom and the "Choosing Wisely" campaign of the American Board of Internal Medicine suggest that imaging should not be performed routinely in patients with back pain, but should be reserved for patients with a suspected serious underlying condition. $5,8,14$

\section{Management of back pain}

Management and treatment of back pain, especially lower back pain, extends from a plethora of either non-pharmacological or pharmacological treatment.

As illustrated in Figure 2, back pain involves non-specific causes and three key areas must be considered for effective management.

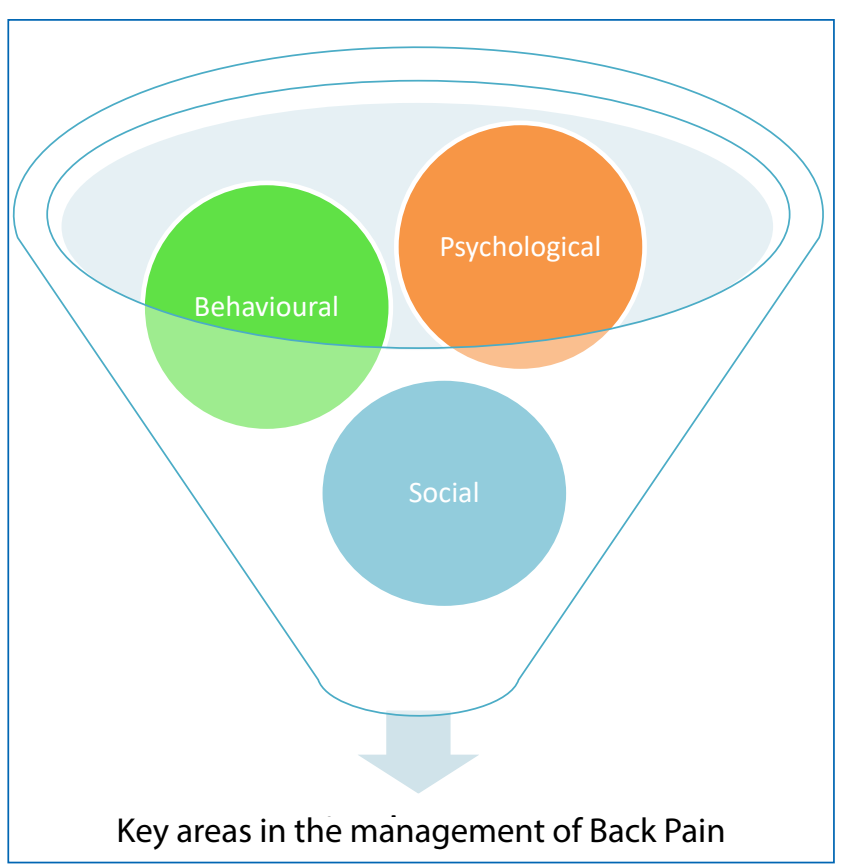

Figure 2: Key areas to consider in the management of back pain

In the past few decades, emphasis on self-management, physical and psychological therapy have taken precedence over pharmacological treatment and surgery. Non-pharmacological methods are the first line and, if unsuccessful, pharmacological treatments are used. ${ }^{16}$

\section{Non-pharmacological treatment}

Exercise, complementary and alternative therapies (such as spinal manipulation, acupuncture, massage and mind-body interventions), psychological therapies (such as cognitive behavioural and operant therapy), physical techniques (traction, ultrasound, transcutaneous electrical nerve stimulation, lowlevel laser therapy, superficial heat or cold and back supports) are some of the non-invasive, non-pharmacological management of back pain. ${ }^{17}$

\section{Exercise}

Exercise is a widely used treatment for back pain. A combination of general physical fitness, aerobic exercise, muscle strengthening and flexibility and stretching exercises are some of the interventions suggested in the treatment of back pain. ${ }^{18}$ Other exercises include traditional mind-body exercises, e.g. Tai 
Chi and yoga, which emphasise precise controlled movement and bodily awareness. However, individual exercises seem to be more beneficial than group exercises in the treatment of back pain. ${ }^{5}$

\section{Spinal Manipulation}

This method provides modest short and long-term relief of back pain, improves psychological well-being and increases functioning. ${ }^{19}$ High velocity thrust techniques, such as mobilisation, have shown to be effective as reported by Maher. ${ }^{5}$

\section{Acupuncture}

Acupuncture is a traditional Chinese-based therapeutic method. To improve painful states, small solid needles are inserted into specific points in the body. ${ }^{20}$ An overview of systematic reviews done by Liu et al. showed that using acupuncture in isolation or as adjunct therapy provided short-term improvements in function and pain for chronic lower back pain. ${ }^{21}$

\section{Pharmacological treatment}

In the treatment of lower back pain, the choice of a suitable drug should be based on the benefits, risks and costs.

\section{Non-opioid analgesics}

The following non-opioid related medicines are available for managing pain in children: paracetamol, and the non-steroidal anti-inflammatory drugs (NSAIDs), for example, naproxen, ibuprofen and mefenamic acid. They adequately treat mild pain and moderate-to-severe pain in combination with other medicines, particularly opioids, to provide more effective relief and reduce adverse effects.

\section{Paracetamol}

Paracetamol is one of the drugs of choice in pain management, due to its excellent safety profile and lack of any significant side-effects. It acts as a prodrug, with an active cannabinoid metabolite. In the brain and spinal cord, paracetamol follows deacetylation to its primary amine ( $p$-aminophenol) which is conjugated with arachidonic acid to form $\mathrm{N}$ arachidonolylphenolamide, a compound known as an endogenous cannabinoid. The involved enzyme is fatty acid amide hydrolase. $\mathrm{N}$-arachidonolylphenolamide is an agonist at the Transient Receptor Potential Cation Channel, Subfamily V, Member 1 (TRPV1) receptors and an inhibitor of cellular anandamide uptake, which leads to increased levels of endogenous cannabinoids, inhibiting cyclooxygenases in the brain at concentrations that are probably not attainable with analgesic dosages of paracetamol. It is of interest to note that a cannabinoid-1 receptor antagonist, given at a dosage level that completely prevents the analgesic activity of a selective cannabinoid receptor agonist, completely inhibits the analgesic activity of paracetamol as well. This fact allows us to explain the mechanism of action of paracetamol in more detail. Despite this finding, however, the definite proof that the analgesic and antipyretic effects of paracetamol are dependent on cyclooxygenase (COX)-inhibition is still unclear. Hence, it works effectively when combined with codeine for more effective control of moderate-to-severe pain and discomfort. ${ }^{22}$

Paracetamol is available orally, in several tablet and liquid formulations, however the dosage should be guided by the age and general condition of the patient.

Paracetamol, was once recommended as the first line treatment for pain due to its low cost. ${ }^{23}$ However, the ineffectiveness of paracetamol in acute lower back pain, hepatotoxicity and the risk of asymptomatic elevation of aminotransferase levels at dosages of $4 \mathrm{~g} / \mathrm{d}$ even in healthy adults poses a potential for harm. ${ }^{16,23}$

\section{Non-steroidal anti-inflammatory drugs}

Non-Steroidal Anti-inflammatory agents (NSAIDs) competitively inhibit the cyclooxygenase (COX) enzyme; the enzymes facilitate the bioconversion of arachidonic acid to inflammatory prostaglandins. This results in the blockade of prostaglandin synthesis and subsequently dampened inflammatory responses. COX-1 and 2 are isozymes that only vary genetically. NSAIDs have three pharmacological preferred attributes, i.e. analgesia, anti-inflammatory and anti-pyretic activity. They generally have similar analgesic properties but selection is based on their receptor selectivity. COX-1 receptor activation produces gastric effects that mediate hyper-secretion of gastric acid, thinning of the lumen and propagate the development of gastric ulcers. These medicines have various formulations. ${ }^{24,25,26}$

The only over-the-counter (OTC) available pain medications are aspirin (S0) and paracetamol (S2) and require no prescription. The NSAID ibuprofen is $\$ 2$ when intended for the treatment of post traumatic conditions, such as pain, swelling and inflammation, for a maximum period of five days without a prescription. All other NSAIDs are S3 and can only be obtained via a prescription from a physician. ${ }^{27}$

It is important to note that NSAIDs have ceiling analgesic effects but the cyclooxygenase-2 mediated anti-inflammatory effects are dose dependant. COX-2 is not detected in most normal tissues, but its expression is rapidly induced by stimuli, such as proinflammatory cytokines (IL-1b, TNFa), lipopolysaccharides, mitogens and oncogenes (phorbol esters), fibroblast growth factor, epidermal growth factor, (luteinizing hormone, LH) and fluid-electrolyte haemostasis, resulting in increased synthesis of PGs in inflamed and neoplastic tissues. ${ }^{24}$

The non-steroidal anti-inflammatory drugs (NSAIDs), such as aspirin, ibuprofen, diclofenac, ketorolac and mefenamic acid, have analgesic and anti-inflammatory properties, which are useful in the management of pain..$^{28}$

Non-selective NSAIDs (ibuprofen) and cyclooxygenase-2 inhibitors, such as celecoxib, have an increased risk of myocardial infarction. ${ }^{23}$ Physicians must take cognisance of these risk factors and when an NSAID or a COX-2 inhibitor is prescribed, the lowest dose for the shortest duration should be considered. ${ }^{16}$ However, in patients over 45 years old and those who have a high risk of gastro-intestinal toxicity, a cost effective proton pump inhibitor should be co-prescribed. ${ }^{29}$ Aspirin and naproxen are the safest 
NSAIDs in high risk cardiovascular patients especially due the anti-platelet properties of aspirin. ${ }^{30}$

\section{Opioid analgesics}

Opioid analgesics will provide analgesia for moderate to severe pain, for the vast majority and with a wide margin of safety. This group includes the following examples: codeine, morphine, oxycodone, methadone, fentanyl and pethidine. Opioids can be divided into weak and strong opioids. Weak opioids are used alone or in combination with other analgesics, in management of moderate pain. Strong opioids are usually reserved for severe pain. ${ }^{1}$

Opioids are derivatives of morphine and bind to opioid receptors. ${ }^{30}$ Their use is contentious and should be judiciously used in selected patients for a short duration and closely monitored due to the risks of addiction and respiratory depression. ${ }^{16,30}$ Tramadol, a weak opioid with some serotonin norepinephrine reuptake inhibitor (SNRI) activity, provides short term improvements in pain and function but data for long-term use is lacking. ${ }^{19}$ Cautionary measures must be taken as serotonin syndrome has been shown to occur when combined with certain antidepressants. ${ }^{30}$

\section{Skeletal muscle relaxants}

Skeletal muscle relaxants are classified into two main categories namely, antispasticity and antispasmodic medications. Antispasticity medications (e.g. baclofen) act on the spinal cord or on the skeletal muscles themselves to better muscle hypertonicity and involuntary spasms. Antispasmodic medications lessen muscle spasms through alterations of central nervous conduction. These agents are divided into benzodiazepines and nonbenzodiazepines. ${ }^{31,32}$

A new skeletal muscle relaxant in South Africa is Myprocam ${ }^{\circledast 3}{ }^{32}$ The active ingredient is cyclobenzaprine, a nonbenzodiazepine antispasmodic agent, which blocks nerve impulses recognised as pain. Cyclobenzaprine is structurally related to the tricyclic antidepressants, like amitriptyline and nortriptyline. It is categorised as a muscle relaxant with a mechanism of action not fully understood, but is thought to be an agonist of the $\alpha 2$ receptor at the descending noradrenergic neurons within the supraspinal area of the brain stem. Some evidence also revealed seretonergic antagonism of the 5 - $\mathrm{HT} 2$ receptors. ${ }^{32,33}$

Myprocam $^{\circledast}$ is often combined with analgesics like ibuprofen or naproxen and is used in addition to rest and physical therapy for short-term relief of muscle spasm associated with acute, painful musculoskeletal conditions. The extended release cyclobenzaprine has shown to have superior efficacy to the placebo in the treatment of lower back pain and has lower frequency of adverse effects compared to other skeletal muscle relaxants. ${ }^{34}$ The recommended adult dose is a $15 \mathrm{mg}$ capsule, taken once daily. Some patients may require up to $30 \mathrm{mg}$ per day, administered as one Myprocam ${ }^{\circledast} 30 \mathrm{mg}$ capsule, taken once daily, or as two Myprocam ${ }^{\circledast} 15 \mathrm{mg}$ capsules, taken once daily. ${ }^{31,32,33}$
Side effects include dizziness and drowsiness. Other anticholinergic effects such as dry mouth, blurred vision, constipation and urinary retention will be expected due to activity on cholinergic receptors. Cardiac arrhythmias like QTC prolongation is likely to arise and should be used with caution in patients with a history of arrhythmias or who are using any medications prolonging the QTc interval. Myprocam ${ }^{\circledR}$ is contraindicated in patients older than 65 years, or in patients with impaired liver function. ${ }^{32}$

Adequate evidence for the effectiveness of the prolonged use of Myprocam ${ }^{\circledast}$ is not available and therapy for longer periods of use is seldom warranted; the duration of use should hence only be for short periods of not more than three weeks. ${ }^{32}$ Skeletal muscle relaxants are used as adjunctive treatment for lower back pain and not considered first line because of the adverse effects such as sedation. ${ }^{30}$

\section{Conclusion}

Back pain can be a debilitating condition that can affect productivity in the workplace. This commonly reported primary healthcare condition requires a thorough patient history to identify the cause and the risk factors for back pain. Risk factors such as depression, physically demanding jobs, insomnia and anxiety play a crucial part in the diagnosis and management of back pain. Non-pharmacological methods such as acupuncture, spinal manipulation and appropriate exercise are valuable first line treatments, and upon failure of these treatments, pharmacological management, such as the use of NSAIDs, opioids and muscle relaxants, should be used when warranted.

\section{References}

1. Baumann TJ, Herndon CM, Strickland JM. Chapter 44: Pain Management In: DiPiro JT, Talbert RL, Yee GC, Matzke GR, Wells BG, Posey L. DiPiro J.T., Talbert R.L., Yee G.C., Matzke G.R., Wells B.G., Posey L Eds. Joseph T. DiPiro, et al.eds. Pharmacotherapy: A Pathophysiologic Approach, ge New York, NY: McGraw-Hill; 2014.

2. Patrick N, Emanski E, Knaub MA. Acute and chronic low back pain. Med Clin North Am. 2014;89:777-89.

3. Hartvigsen J, Hancock MJ, Kongsted A, Louw Q, Ferreira ML, Genevay S, et al. What low back pain is and why we need to pay attention. Lancet. 2018;391:2356-67.

4. Steffens $D$, Ferreira ML, Latimer J, Ferreira PH, Koes BW, Blyth F, et al. What triggers an episode of acute low back pain? A case-crossover study. Arthritis Care Res. 2015;67:403-10.

5. Maher C, Underwood M, Buchbinder R. Non-specific low back pain. Lancet. 2017;389:736-47.

6. Pillastrini P, Gardenghi I, Bonetti F, Capra F, Guccione A, Mugnai R, Violante FS. An updated overview of clinical guidelines for chronic low back pain management in primary care. Joint Bone Spine. 2012;79:176-85.

7. Chou L, Brady SRE, Urquhart DM, Teichtahl AJ, Cicuttini FM, Pasco JA, et al. The association between obesity and low back pain and disability is affected by mood disorders: A population-based, cross-sectional study of men. Medicine. 2016;95(15):e3367.7p

8. Golob AL, Wipf JE. Low Back Pain. Med Clin North Am. 2014;98:405-28.

9. Agmon $M$, Armon $G$. Increased insomnia symptoms predict the onset of back pain among employed adults. PLoS ONE. 2014;9(8):e103591.7p.

10. Hoffman BM, Papas RK, Chatkoff DK, Kerns RD. Meta-analysis of psychological interventions for chronic low back pain. Health Psychol. 2007;26(1):1-9.

11. Kamper SJ, Apeldoorn AT, Chiarotto A, Smeets RJEM, Ostelo RWJG, Guzman J, et al. Multidisciplinary biopsychosocial rehabilitation for chronic low back pain Cochrane Syst Rev and Meta-analysis. BMJ. 2015;350:h444. 11p. 
12. Saper RB, Sherman KJ, Delitto A, Herman PM, Stevans J, Paris R, et al. Yoga vs. physical therapy vs. education for chronic low back pain in predominantly minority populations: study protocol for a randomized controlled trial. Trials. 2014;15:67.21p.

13. Rigoard P, Blond S, David R, Mertens P. Pathophysiological characterisation of back pain generators in failed back surgery syndrome (part B). Neurochirurgie. 2015;61:S35-S44.

14. Wheeler SG, Wipf JE, Staiger TO, Deyo RA, Jarvik, JG. Evaluation of low back pain in adults. UpToDate. 2018 [accessed on 26 June 2018];Topic 7782,Version 69.0. Available at: https://www.uptodate.com/contents/ evaluation-of-low-back-pain-in-adults.

15. Herndon CM, Zoberi KS, Gardner BJ. Common question about chronic low back pain. Am Fam Physician. 2015;91(10):708-14.

16. Foster NE, Anema JR, Cherkin D, Chou R, Cohen SP, Gross DP, et al. Prevention and treatment of low back pain: evidence, challenges, and promising directions. Lancet. 2018;391:2368-83.

17. Chou R, Deyo R, Friedly J, Skelly A, Hashimoto R, Weimer M, et al. Nonpharmacologic therapies for low back pain: a systematic review for an American College of Physicians Clinical Practice Guideline. Ann Intern Med. 2017;166(7):493-505.

18. Hayden J, Van Tulder MW, Malmivaara A, Koes BW. Exercise therapy for treatment of non-specific low back pain. Cochrane Libr. 2005.

19. Last AR, Hulbert K. Chronic low back pain: evaluation and management. Am Fam Physician. 15 Jun 2009;79(12):1067-74.

20. Mogole O, Kandiwa R, Babarinde O, Ismail H, Dlamini N, Maluleke L, et al. Muscle pain. S Afr Fam Pract. 2017;59(3):24-32.

21. Liu L, Skinner M, McDonough S, Mabire L, Baxter GD. Acupuncture for low back pain: an overview of systematic reviews. Evid Based Complement Alternat Med. $2015 ; 2015$.
22. Ottani A, Leone $S$, Maurizio S, Ferrari A, Bertolini A. The analgesic activity of paracetamol is prevented by the blockade of cannabinoid CB1 receptors. Eur J Pharmacol. 2006;531(1):280-1.

23. Chou R, Qaseem A, Snow V, Casey D, Cross JT, Shekelle P, Owens DK. Diagnosis and treatment of low back pain: a joint clinical practice guideline from the American College of Physicians and the American Pain Society. Ann Intern Med. 2007;147(7):478-91.

24. Zarghi A, Arfaei S. Selective COX-2 Inhibitors: A Review if Their Structure Activity Relationships. IJPR. 2011 [accessed on 13 June 2018];10(4). Available at: https:// www.ncbi.nlm.nih.gov/pmc/articles/PMC3813081/

25. Chan AT, Dettering E. Prospects For Chemoprevention Of Colorectal Neoplasia. 1st ed. Berlin: Springer, 2013.

26. Park HJ, Dong EM. Pharmacologic Management Of Chronic Pain. Korean J Pain. 2010;23(2):99-108.

27. Medicines and related substance Act 101 of 1965. Accessed on 16 June 2018 Available at: http://www.hpcsa.co.za/Uploads/editor/UserFiles/downloads/ legislations/acts/medicines_and_related_sub_act_101_of_1965.pdf

28. Lundgren C, Mohr W. SA Acute Pain Guidelines. S Afr J Anaesth. 2009;15(6).

29. Savigny $P$, Watson $P$, Underwood M. Early management of persistent non-specific low back pain: summary of NICE guidance. BMJ (Online). 2009;338.

30. Chou R. Pharmacological management of low back pain. Drugs. 1 Mar 2010;70(4):387-402.

31. Witenko C, Mooman-Li R, Motycka C, Duane K, Hincapie-Castillo J, Leonard P, Valaer $C$. Considerations for the appropriate use of skeletal muscle relaxants for the management of acute low back pain. J Pharm Ther. 2014;39(6):427-35.

32. Myprocam ${ }^{\circledast}$. South African package insert. March 2014.

33. Truven Health Analytics. Cyclobenzaprine Drugdex monograph. Micromedex Online, 2015.

34. Weil AJ. Cyclobenzaprine extended release for acute low back and neck pain. Therapy. 2009;6(6):871-81. 